\title{
Discussion on papers published in the
}

\author{
Magazine of Concrete Research
}

Volume 35, Number 122 : March 1983

\section{Bimoment design of some rectangular slabs*}

\author{
Colin R. Gurley \\ Contribution by C. T. Morley \\ University of Cambridge, Department of Engineering
}

Mr Gurley is to be congratulated on another interesting paper, demonstrating that quite complicated torsionless-grillage problems can be tackled successfully by his bimoment method. His sensible use of a small number of bands of reinforcement, or sets of strips, with the same moment pattern in every strip in a band, permits the bimoment equation to be deployed to great advantage. In some problems, e.g. Figure 15, this sensible choice of bands would also permit a rapid solution by considering strip moment patterns only, without using the bimoment equation.

His example solutions seem to be based on another valuable insight, that consideration of likely collapse mechanisms can help a designer to see where extra reinforcement (above the Code minimum) will do the most good (an insight consistent with other work ${ }^{(1)}$ on design with variable reinforcement, where optimal designs collapse in a special type of mechanism).

Some of the solutions are 'exact', with equilibrium moment field and a consistent collapse mechanism, for a torsionless grillage. However, the significant torsional strength ${ }^{(2)}$, even for very low concrete tensile strength, of real slabs with orthogonal reinforcement, makes it unlikely that collapse mechanisms involving 'hypars' will be observed in experiments. Real slabs, even with variable reinforcement,

\footnotetext{
*Pages 3-18 of MCR 122.
}

tend to collapse in mechanisms approximating to the Johansen type, with straight yield lines between 'rigid' regions. On cracking, torsional stiffness certainly decreases more than bending stiffness does, to around $20 \%$ of the post-cracking bending stiffness for slabs with steel at one face only - a significant but perhaps not drastic reduction, affecting serviceability rather than strength. The Hillerborg assumption of zero twisting moments has the very great merit of leading to a simple lower-bound design method. We should perhaps discuss to what extent, and in what circumstances, the advantages of thinking of torsionless grillages outweigh the possible economic penalty inherent in neglecting the torsional strength also provided by the reinforcement.

The torsional strength of a real slab can significantly increase its collapse load over that of the corresponding torsionless grillage. Consider, for example, a simply supported square slab of $\operatorname{span} L$, corners held down, with uniform load $p$ and uniform top and bottom reinforcement in both directions giving ultimate moment $m$ per unit width. The torsionless grillage would collapse at $p L^{2}=16 \mathrm{~m}$, in a mechanism involving four hypars. If torsional strength is included (i.e. if the familiar Johansen yield criterion is adopted, with ultimate moment $m$ on a yield line in any direction), the 'exact' plastic collapse load $^{(3)}$ is $p L^{2}=24 m$, an increase of $50 \%$. (The collapse mechanism had diagonal yield lines separating 
undeforming portions.) If there is no top reinforcement, and the corners are not held down, the torsionless grillage still collapses at $p L^{2}=16 \mathrm{~m}$. For the slab with torsional strength ${ }^{(2)}$ a lower-bound approach (with the twisting moment $M_{x y}$ constant on hyperbolae within each quarter of each quadrant of the slab) gives $p L^{2}=17.45 \mathrm{~m}$, a collapse load increase of at least $9 \%$.

\section{Reply by the author}

(1) I thank Dr Morley for his kind comments. His opening paragraphs raise issues which seem applicable to any form of structural design whatsoever.

It is natural and commonplace for designers to simplify structural problems by making some specific assumption(s) about stresses or stress-resultants so as to reduce the degree of static indeterminacy. Any such approach necessarily relies on the lower-bound theorem of plasticity, i.e. that which states that any statically correct equilibrium solution can be carried through to a safe design, provided only that there is sufficient ductility. The point that now seems simple and obvious, even if not always explicitly made, is that any well formulated static assumption will always have certain quite specific consistent kinematic implications. If one assumes zero moment at some definite location in an indeterminate beam problem, then one has obviously inserted a hinge at that point.

If we must simplify, then surely it is sensible to carry the process to its logical conclusion - i.e. to utilize both upper- and lower-bound theorems by seeking a collapse mechanism which is consistent with the original simplifying assumption. It seems obvious that solutions obtained on this basis will always be more economical than those based solely on the lower-bound theorem, i.e. without any explicit consideration of mechanism compatibility. It also seems obvious that a designer who has a correct appreciation of the critical collapse mechanism will do a better job than one who does not. Clarity of understanding is a major safeguard against inappropriate designs and gross error.

(2) I also have found Rozvany's work tantalizing and it would indeed be interesting to try to reconcile his methods with my own in the quite large areas where they overlap. Unfortunately those who would read Rozvany in close detail need to have invested much more time in background mathematical material than most practising designers (myself included) can afford.

(3) The effect of the post-cracking reduction of torsional stiffness upon serviceability seems important to me, even though 'elastic' designers have been neglecting it for decades. Certainly the

\section{REFERENCES}

1. Rozvany, G. I. N. Optimal design of flexural systems. Oxford, Pergamon Press, 1976, pp. 297. (See especially pp. 245-248.)

2. NIELSEN, M. P. The theory of plasticity for r.c. slabs. IABSE Colloquium on Plasticity in Reinforced Concrete, Copenhagen 1979. Introductory Report, Zurich. 1979. pp. 93-114. (See p. 96.)

3. WOOD, R. H. Plastic and elastic design of slabs and plates. London, Thames \& Hudson, 1961. pp. 344. (See p. 88.)

Australian profession has had many serviceability problems with flat plates which, whilst not often dramatic, have had widespread nuisance value. Australia does seem to utilize very many long-span (6 to $10 \mathrm{~m}$ ) flat plates. Perhaps European experience is different because the spans are not usually so long.

(4) Certainly the motivation for a torsion-free (Hillerborg's 'Simple Method') approach is pragmatic and my own work merely attempts to optimize the possibilities of that approach by incorporating consistent upper-bound (mechanism) considerations into it. The Johansen yield-line approach has been around for more than fifty years now and it has been very widely disseminated in the English-language technical press. One has great regard for Johansen and for his successors at Copenhagen, but the fact is that, among English-speaking practitioners, Johansen analysis is still used only rarely and by a minority.

Bimoment methods have been around for about five years and I am confident that, as they develop further over, say, the next five years, they will come to provide clear, straightforward and economic solutions to the main bulk of routine design problems.

There is certainly a cost penalty, but the example chosen by Dr Morley is an extreme case. If one makes comparisons involving simple 'uniform top and bottom reinforcement', the penalty can be substantial. But, of course, Johansen methods cannot easily address problems in which even a modest degree of optimization of the reinforcement layout is attempted. Bimoment methods can address such problems much more easily. Dr Morley's 50\% penalty assumes uniform top steel equal to bottom steel throughout a simply supported slab. But, of course, the bimoment solution does not require any top steel at all for strength, although I would usually provide some localized corner top steel for serviceability. A 9\% penalty is closer to the truth and may be acceptable to many designers. Hillerborg has shown that, by going to just one strong central band in each direction, the penalty can be reduced to less than $1 \%$.

A more dramatic example against bimoment methods would consider a square slab simply sup- 
ported on two adjacent edges and unsupported on the other two. If the reinforcement is run parallel to the supports, then any torsion-free analysis will conclude that the collapse strength is zero regardless of reinforcement content. Practitioners may like to consider whether they would be prepared to stake their reputations on any such design without making very careful estimates of service load performance. Surely it would be much more appropriate to place the reinforcement in diagonal directions? Such a reinforcement array would require further development of bimoment methods than so far published, but it can be done in a reasonably straightforward way. One hesitates to generalize, but I do suspect that cases in which the cost penalty (for a bimoment design in comparison with a Johansen design) is large are cases which are suspect on serviceability grounds. And, of course, both methods are usually much more economic than any other accepted approach.

(5) I suggest that the need of the practising profession is for a method which can be applied fairly universally with reasonable clarity and reasonable economy over the very wide range of problems arising in design practice. We cannot limit our clients to those problems dealt with in handbooks and we cannot spend unlimited time on literature searches. I suggest that the bimoment approach offers the best available promise. The next five years should see greater clarity, greater simplicity and greater universality in the application of these methods. 